\title{
Fabrication and Characterization of Nanoporous Organically Modified Silica Films on Electrodes as Platforms for Electrocatalytic Oxidation of Phospholipids
}

\author{
Layla B. Mehdi ${ }^{1,2}$, Nigel Browning ${ }^{2}$ and James A. Cox ${ }^{1}$ \\ ${ }^{1}$ Miami University, Chemistry Department, Oxford, OH, 45056 \\ ${ }^{2}$ Material Science Division, Pacific Northwest National Laboratory, Richland, WA, 99352
}

Phospholipids, PLs, are major and dynamic constituents of the cellular membrane. They play an active role in basic mitochondrial functions, sensing, transducing, prolongation and apoptosis processes [1]. In this project the main focus was fabrication of electrodes modified with nanoporous organically modified sol-gel (ormosil) films for application to the electrocatalytic detection at of two major phospholipids, cardiolipin (CL) and phosphatidylcholine (PC).

The occurrence of $\mathrm{CL}$ is strictly localized in the mitochondrial inner membrane. It takes part in regulation of the permeability of the mitochondrial membranes upon reception of apoptotic proteins [2]. Monitoring the concentration of $\mathrm{CL}$ is important because a decrease is related to an increased amount of cytochrome $\mathrm{c}$ released during the apoptosis process [3]. In contrast to $\mathrm{CL}, \mathrm{PC}$ is an abundant phospholipid that is a part of all organelles membranes. PC also acts as a source in the fabrication of secondary messengers that alter cell growth, such as phosphatidic acid and diacylglycerol. The decrease in PC level affects cell growth and synthesis of cellular membranes [4]. In this context, decreased amounts of $\mathrm{CL}$ and $\mathrm{PC}$ in mitochondria is an initial step in the apoptosis process and in conditions related to their level, such as rheumatoid arthritis, heart failure, and Barth Syndrome [5].

Electrocatalytic detection of phospholipids is complicated by the adsorption of products of their electrode reactions or of matrix components, both of which passivate the surface [6]. To address this problem we explored the modification of electrode surfaces by electrochemically-assisted deposition of size-exclusion, nanoscale films comprising organically modified sol-gels (50-100 nm thickness) with 50-60 nm template pores. The film precursor was methoxytrimethylsilane, and the templating agent was 50 -nm (polystyrene sulfonate), PSS.

The electrode surface was first modified with aminopropyltriethoxysilane (APTES) to obtain positive sites. Next, PSS nanobeads with a 50nm diameter were electrostatically attached (Fig. 1A). After ormosil film formation in the zone surrounding these beads, the PSS was dissolved, and the surface was characterized by scanning electron microscopy (Fig. 1B). Of particular interest was whether the array of the resulting pores was varied in terms of density by changing the surface coverage of APTES.

Immobilization of an electrochemical oxidation catalyst, such as ruthenium oxide or dirhodiumsubstituted polyoxometalate-protected gold nanoparticles, $\mathrm{Rh}_{2} \mathrm{PMo}_{11}$-AuNP (Fig. $2 \mathrm{~A}$ and $2 \mathrm{~B}$ ), in the pores of the ormosil was employed for the oxidation of cysteine, methionine, and phospholipids. Successful electrocatalytical activity was demonstrated. Of importance was that the design objective; mitigation of passivation by adsorption was accomplished. For example, the cyclic voltammetry peak current for the reduction of hexacyanoferrate was measured in the presence and absence of a known surface active agent, PC (which was itself not electroactive under the conditions used). The data demonstrate that $300 \mu \mathrm{M}$ PC did not lower the peak current by a statistically significant amount. 
References:

[1] L. J. Pike, J. Lipid Res., 2003, 44, 655

[2] E. K. Tuominen, C. J. Wallace, P. K. Kinnunen, J. Biol. Chem., 2002, 277, 8822

[3] D. B. Ostrander, G. C. Sparagna, A. Amoscato, J. McMillin, J. Biol. Chem., 2001, 276, 38061

[4] V. Zaremberg, C. R. McMaster, J. Biol. Chem., 2002, 277, 39035

[5] M. M. Wright, A. G. Howe, V. Zaremberg, Biochem. Cell Biol. 2004, 82, 18

[6] M. Wandstrat, J.A. Cox, W. U. Spendel, G. E. Pacey, Electroanalysis 19, 139 (2007)

A portion of this work was performed at the Pacific Northwest National Laboratory, which is operated by Battelle Memorial Institute for the U.S. Department of Energy under No. DE-AC05-76RL01830.
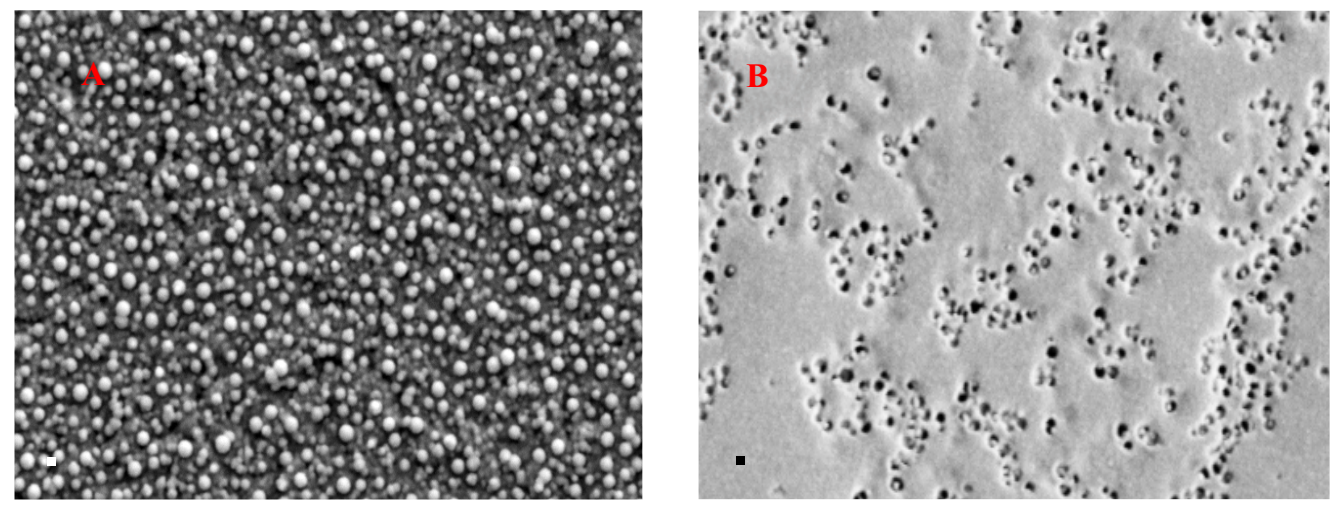

Figure 1. SEM images of glassy carbon surfaces modified with PSS $0.001 \mathrm{M}$ (A) and resulting nanopores ormosil film (B). The scale bars; $200 \mathrm{~nm}$.
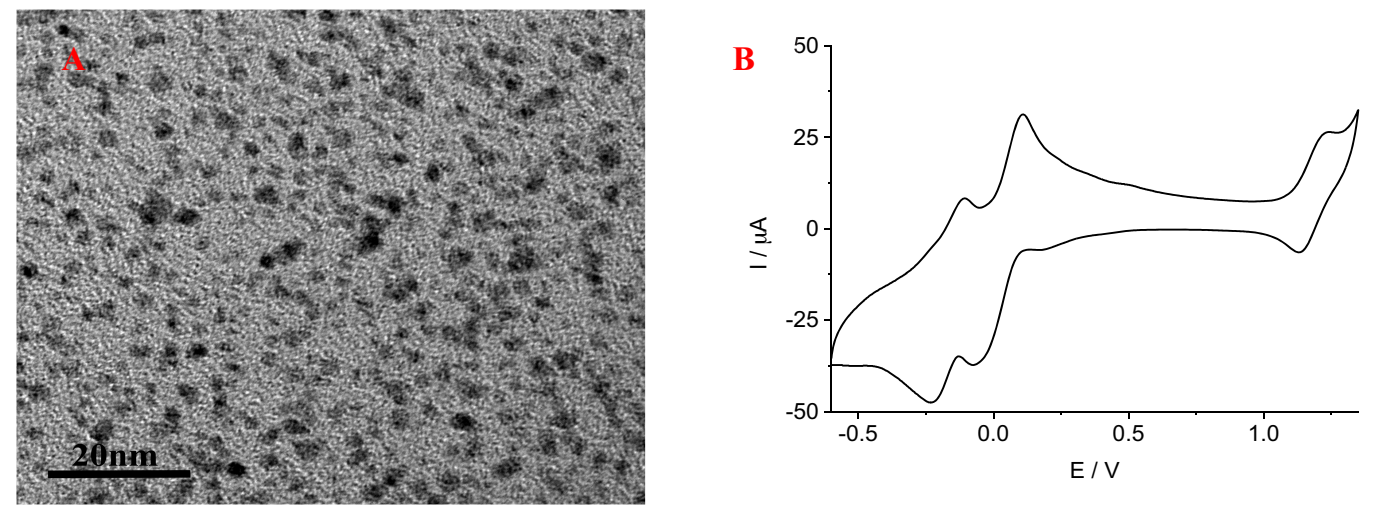

Figure 2. (A) TEM of $\mathrm{Rh}_{2} \mathrm{PMo}_{11}-\mathrm{AuNP}\left(\mathrm{d}_{\text {mean }}=3.0 \pm 1.0 \mathrm{~nm}\right)$ and (B) cyclic voltammetry of $\mathrm{Rh}_{2} \mathrm{PMo}_{11^{-}}$ AuNP catalyst immobilized at nanopores ormosil film in $0.5 \mathrm{M} \mathrm{H}_{2} \mathrm{SO}_{4}$. 